\title{
On Sensory Terminations in Oral Cavity Base in Man
}

By

\author{
Masao Ohgaki and Koya Hotta \\ (大垣政男) (堀田康哉)
}

(From the Anatomical Laboratory of Prof. H. Seto, Faculty

of Medicine, Tohoku University, Sendai)

(Received for publication, August 28, 1952)

As far as we are aware, there has been no histological study reported hitherto on the innervation of the mucous membrane of the base of the oral cavity, either of man or mammals. One of the authors of this report, Ohgaki, ${ }^{11}$ previously conducted a minute study on the innervation of the under surface of human tongue, utilizing microscopic slides prepared by Seto's silver impregnation, and succeeded in obtaining many new informations. In succession to the above, we jointly made a strenuous research on the innervation of the basal surface of the oral cavity, using a series of slides similarly prepared and stained, and arrived at the following results.

As the mucous membrane of the oral cavity base is posteriorly containuous with that of the under surface of the tongue, the histological image of the former resembles strongly that of the latter. Namely, the epithelium is composed of the same low non-cornified stratified flat epithelium in general, and the papillae formation of the connective tissue of lamina propria against the epithelium is generally very poor. Accordingly, the development of nerves, especially sensory nerves must be also weak in this region. That is to say, it would be quite easy to conclude, from the above mentioned histological image, the poor development of the sensory nerves in the mucous membrane of this area.

One of us, Ohgaki, in his previous study succeeded in discovering a rather large number of his so-called taste-bud papillae in the epithelium of the under surface of the tongue. So we undertook a forced scrutiny of the mucous membrane of the oral cavity base, which is similar histologically with the under surface of the tongue, for similar papillae, but we found that, generally speaking, this part does not contain taste-bud papillae, because we encountered such tast-buds only in almost negligible few cases. They are formed in usual one or two in the in particular thinned out epithelium which is caused by the tip of a small papilla juttiug 
out very strongly into the epithelium, as shown in Fig. 4. After all, only a very few weakly developed taste-buds are observable here.

Now to turn to the innervation of the mucous membrane of the oral cavity base. The small nerve bundles reaching the lamina submucosa are themselves very few in number, so the plexus formed in this part is also of extremely weak development. The nerve elements consist of fine non-medullated vegetative and thick medullated sensory nerve fibres, the former spreading out in the connective tissue beneath the epithelium to terminate as the Stöhr's vegetative terminalreticulum, standing in tactile innervation for small blood vessels, blood capillaries, connective tissue cells etc.

The sensory nerve fibres rise up through the connective tissue beneath the epithelium, most of them running into the papillae, to terminate in their upper parts. But the number of sensory fibres is so small that such papillae as not provided with sensory innervation are found numerously, that is, only a small minority of papillae contains sensory fibres. Consequently, the mucous membrane of the oral cavity base is much poorer in sensory innervation than the under surface of the tongue.

Furthermore, the terminal formation of the sensory nerve fibres is

Fig. 1

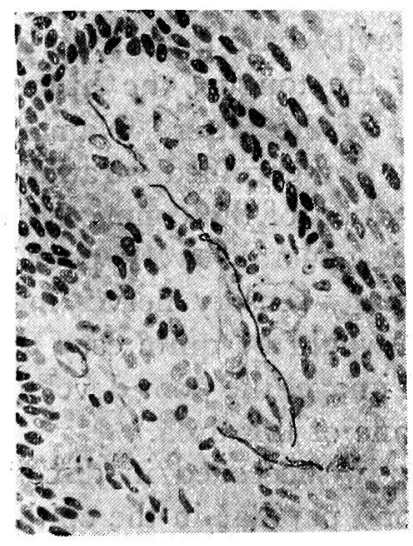

Fig. 2

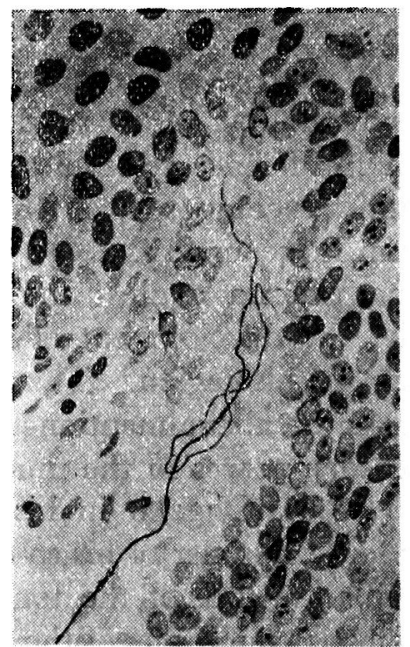

Fig. 1. An unbranched termination found in a small papilla. Mucous membrane of oral cavity base in man. Seto's silver method, $\times 500$, reduced to $2 / 3$.

Fig. 2. An simple branched termination found in a small papilla in mucous membrane of oral cavity base in man. Same staining, $\times 700$, reduced to $2 / 3$. 
formed much simpler than that in the under surface of the tongue, the majority being represented as unbranched terminations consisting of minute fibres, running a simple course and terminating sharply right under the epithelium in the tip of the papillae, as shown in Fig. 1. However, there are often seen simple branched terminations of more complex constitution, but they are also composed of fine fibres showing a very simple course and terminating in general sharply in the tip of the papillae (Fig. 2).

As described above, the sensory nerve terminations in the mucous membrane of the oral cavity base are in general of very simple conformation and very small in quantity also, but in very rare cases, some medium sized medullated fibres, upon penetrating the papilla, after losing their myelin and branching out into two or three rami, go over into the glomerular termination, though formed very simply. Fig. 3 shows a typical glomerular termination, from which one nerve fibre is seen running further up to the close vicinity of the epithelium, to terminate sharply. The nerve elements concerning this glomerular formation often show more or less change in size.

In the oral cavity base also were not observed such intraepithelial

Fig. 3

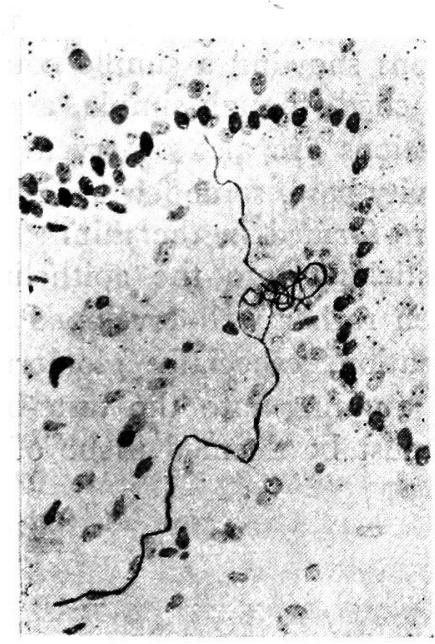

Fig. 4

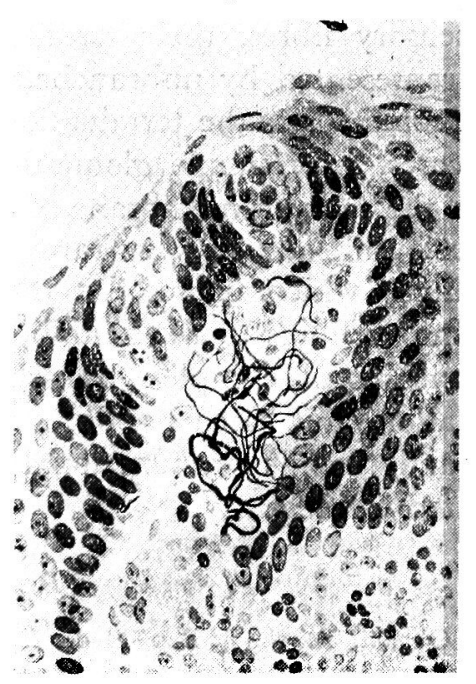

Fig. 3. A very simple glomerular termination formed in a small papilla. Mucous. membrane of oral cavity base in man. Same staining, $\times 500$, reduced to $2 / 3$.

Fig. 4. A glomerular termination formed beneath two taste-buds found in the thin epithelium covering the tip of a small papilla. Mucous membrane of oral cavity base in man. Same staining, $\times 500$, reduced to $2 / 3$. 
fibres coming from the sensory terminations described above into the epithelium.

It was imagined that the taste-buds found very rarely in the epithelium of the mucous membrane of the oral cavity base would be of degenerative formations as the majority of those found in the under surface of the tongue, and the development of the sensory nerve fibres for these tastebuds thereto might be very poor. However, it is a remarkable fact that the development of the sensory fibres running towards the taste-buds in this region is often far from poor. As shown in Fig. 4, in the connective tissue directly under two taste-buds a comparatively complex branched termination, especially a quasi-glomerular termination is formed by thick nerve fibres. But, as it seems to be common that also here no existence of intra- and extra-gemmal fibres is observed, these taste-buds are also not of typically sufficient development, and thought to be at least functionally inadequate entities.

\section{SUMMARY}

The development of sensory nerve fibres in the mucous membrane of the oral cavity base is very weak, in parallel with the poor formation of epithelium and papillae in this region, most of the papillae lacking sensory fibres penetrating them. In cases when the papillae are provided with sensory fibres, their terminal formation is generally very simple, being represented by unbranched terminations showing a simple course. But in some cases, the țerminations are branched, though simple, and in rarer cases, very simple glomerular terminations are also observed.

In the mucous membrane of the oral cavity base, some few taste-buds can be found, though very rarely. They are formed in the thinned out epithelium, which caused by the papilla sticking into the epithelium. The sensory fibres to the taste-buds are often rather well-developed and sometimes form complex branched termination, especially glomerular termination, but since no fibres are found running into the taste-buds or around them, their functional capacity must be deemed to be of degenerated inadequacy.

\section{References}

1) Ohgaki, Tohoku J. Exp. Med., 1952, 56, 169. 